\title{
Inertially confined plasma in an imploding bubble
}

\author{
David J. Flannigan and Kenneth S. Suslick *
}

\begin{abstract}
Models of spherical supersonic bubble implosion in cavitating liquids predict that it could generate temperatures and densities sufficient to drive thermonuclear fusion ${ }^{1,2}$. Convincing evidence for fusion is yet to be shown, but the transient conditions generated by acoustic cavitation are certainly extreme ${ }^{3-5}$. There is, however, a remarkable lack of observable data on the conditions created during bubble collapse. Only recently has strong evidence of plasma formation been obtained ${ }^{6}$. Here we determine the plasma electron density, ion-broadening parameter and degree of ionization during single-bubble sonoluminescence as a function of acoustic driving pressure. We find that the electron density can be controlled over four orders of magnitude and exceed $10^{21} \mathrm{~cm}^{-3}-$ comparable to the densities produced in laser-driven fusion experiments ${ }^{7}-$ with effective plasma temperatures ranging from 7,000 to more than $16,000 \mathrm{~K}$. At the highest acoustic driving force, we find that neutral Ar emission lines no longer provide an accurate measure of the conditions in the plasma. By accounting for the temporal profile of the sonoluminescence pulse and the potential optical opacity of the plasma, our results suggest that the ultimate conditions generated inside a collapsing bubble may far exceed those determined from emission from the transparent outer region of the light-emitting volume.
\end{abstract}

A bubble acoustically driven into nonlinear radial oscillation can focus the diffuse energy of the sound field by many orders of magnitude ${ }^{8}$. The energy focusing is such that broadband light emission is observed (sonoluminescence) ${ }^{4}$ and molecular bonds are broken (sonochemistry) ${ }^{9}$. Measurement of the bubble dynamics of a single sonoluminescing bubble (single-bubble sonoluminescence (SBSL)) has shown the implosion velocity to be greater than the speed of sound with enormous acceleration near maximum collapse ${ }^{10}$. The bubble dynamics and the properties of the emitted light suggest the generation of extreme intracavity conditions. Indeed, recent molecular dynamics simulations predict temperatures approaching $10^{8} \mathrm{~K}$ but lasting for only a few hundred femtoseconds ${ }^{2}$. The extreme conditions generated during SBSL arise from quasi-adiabatic compression of the bubble contents. One measure of the intensity of bubble implosion is the ratio of maximum to minimum bubble volume (that is, compression ratio). The value of the compression ratio, and hence the bubble kinetic energy, increases with increasing acoustic pressure $\left(P_{a} ;\right.$ ref. 11). Thus, at high $P_{\mathrm{a}}$ there is more energy available to be transferred to the bubble contents, which should ultimately produce more extreme intracavity conditions.

Recently, Taleyarkhan and co-workers claimed to observe neutrons during acoustic cavitation in deuterated acetone ${ }^{12,13}$ resulting from intracavity fusion reactions (that is, 'sonofusion'). These reports were met with immediate skepticism, and serious issues with the validity of the claims arose $\mathrm{e}^{14,15}$. Indeed, subsequent studies in several independent laboratories have not succeeded in reproducing the original report ${ }^{16-18}$. Despite the controversy surrounding sonofusion, there is no dispute that an imploding bubble can generate high temperatures and pressures. Our spectroscopic work on SBSL in sulphuric acid $\left(\mathrm{H}_{2} \mathrm{SO}_{4}\right)$ quantified heavy-particle temperatures and pressures in excess of $15,000 \mathrm{~K}$ and 4,000 atm, respectively ${ }^{19,20}$. Furthermore, the direct observation of noble-gas ion emission lines (for example, $\mathrm{Xe}^{+}, \mathrm{Kr}^{+}$and $\mathrm{Ar}^{+}$) provided definitive evidence for generation of plasma during cavitational collapse ${ }^{6}$. The critical intracavity plasma properties (that is electron density, ion-broadening parameter and degree of ionization) and their relationship with the dynamics of bubble collapse (as controlled by acoustic driving pressure), however, have not been previously determined. Knowing how the intracavity plasma properties are affected by the bubble's kinetic energy will illuminate the controlling parameters of cavitation as a mechanism for extreme energy processes.

The ideal liquid for cavitation should have both a very low vapour pressure and the ability to solubilize any sonochemical products: otherwise the interior of the bubble becomes rapidly poisoned by polyatomics that sap effective compressional heating by low polytropic $\left(C_{\mathrm{p}} / C_{\mathrm{v}}\right)$ ratios and by endothermic bond dissociation ${ }^{21,22}$. Exotic media such as mineral acids, roomtemperature ionic liquids and molten salt eutectics are therefore attractive for optimizing the extreme conditions generated during cavitation $^{23}$. In this letter, we have spectroscopically analysed the light emitted from a single acoustically driven bubble as a function of acoustic driving force, $P_{\mathrm{a}}$, for SBSL in concentrated sulphuric acid ( $85 \mathrm{wt} \% \mathrm{H}_{2} \mathrm{SO}_{4}$, vapour pressure $=0.04$ torr at $300 \mathrm{~K}$ ) containing Ar at 5\% of saturation. Figure 1 shows a typical emission spectrum along with an image of the acoustic resonator containing a brightly luminescing bubble at its centre (that is, the acoustic field velocity node). The spectrum consists of lines from electronically excited Ar atoms and a featureless continuum attributed to radiative plasma processes (for example, bremsstrahlung, recombination and so on; ref. 24). The emission lines are from electronic transitions between states within the $4 p$ and $4 s$ array, the energies of which range from 11.5 to nearly $14 \mathrm{eV}$. By measuring the relative intensities of the lines, we are able to determine the temperature of the environment from which the Ar atoms are radiating (Fig. 1b, inset).

Figure 2 shows the spectral shape of an isolated Ar line as a function of $P_{\mathrm{a}}$. As can be seen, the line becomes increasingly broadened, redshifted and asymmetric as the driving pressure is increased. Broadening and shifting of emission lines reflect both the rate of impact between the radiating atom and other particles (pressure broadening) and the increase in the magnitude of interaction between the fields of neutral (dispersion forces) and charged (Stark effect) particles and the atomic energy levels ${ }^{25}$. For most processes, the line shape is symmetric, with either a Lorentzian or Gaussian profile. Broadening by fields, however, introduces asymmetries into the profiles, as predicted by perturbation theory of second-order Stark effects $^{25}$. As the energies associated with second-order Stark effects scale as $r^{-4}$, where $r$ is the separation between the ion or the electron 


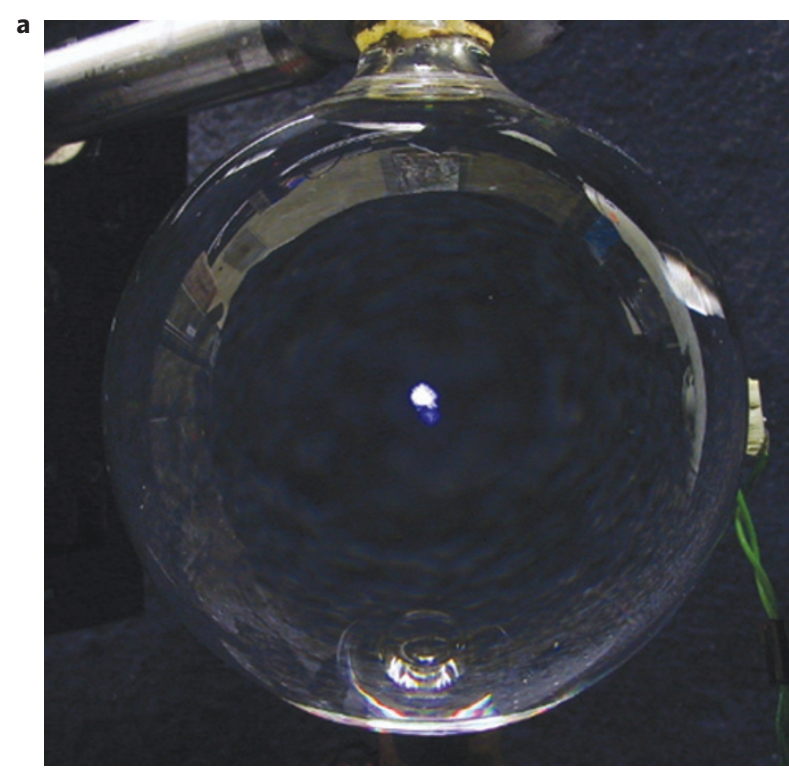

b

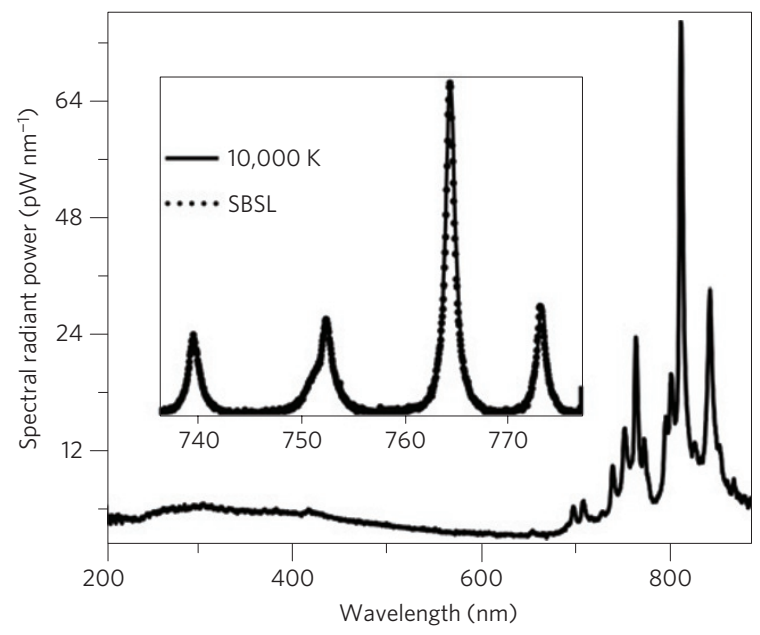

Figure 1 | SBSL from sulphuric acid $\left(85 \mathrm{wt} \% \mathrm{H}_{2} \mathrm{SO}_{4}\right.$ containing $\mathrm{Ar}$ at $5 \%$ of saturation). a, Photograph of a rapidly translating sonoluminescing bubble at the velocity node of a spherical quartz resonator. The driver piezoceramic is partially visible at the bottom of the image, and the microphone is to the right. The entire apparatus is rigidly clamped at the narrow neck of the quartz flask (top of image). $\mathbf{b}$, A typical SBSL emission spectrum from a bubble driven with a relatively low acoustic driving pressure, $P_{\mathrm{a}}$. The emission lines $(\sim 700-900 \mathrm{~nm})$ are due to electronic transitions between states within the $4 p$ and $4 s$ array of neutral Ar. Inset: A higher-resolution spectrum of SBSL Ar emission and a least-squares Lorentzian fit at a thermally equilibrated temperature of $10,000 \mathrm{~K}$.

and the radiator, the effect is most pronounced for highly polarizable atoms in dense ionized gases. In comparison, perturbation by dispersion forces scales only as $r^{-6}$. The magnitude of interaction therefore decreases with distance more rapidly for neutrals than for ions. If ion motion is negligible during the relaxation time of the radiator (as is typically the case for heavier elements) then their motion can be approximated as 'quasi-static'. Within this approximation, line asymmetry depends on two dimensionless parameters: the ion-broadening parameter $(A)$, which is a measure of the relative importance of heavy-ion broadening to electron broadening, and, to a lesser extent, the Debye shielding parameter $(R)$, which reflects the shielding of heavy-ion perturbation by plasma electrons ${ }^{25}$.

The asymmetry in SBSL Ar atom emission lines can be isolated and visualized by comparison with a least-squares-fit Lorenztian

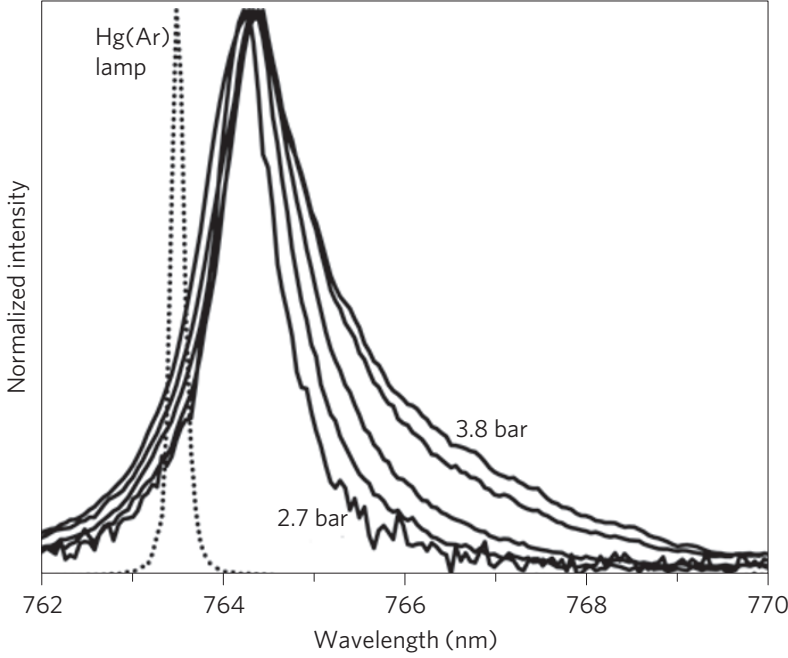

Figure 2 | SBSL Ar emission line profiles as a function of the acoustic driving pressure, $\boldsymbol{P}_{\mathrm{a}}$. The solid lines, from the narrowest linewidth to the broadest, correspond to $P_{a}$ of 2.7, 3.0, 3.3, 3.6 and 3.8 bar. The dotted line centred at $763.51 \mathrm{~nm}$ is the same Ar line $\left(4 p^{2}[3 / 2]\right.$ to $4 s^{2}[3 / 2]$ states) from a low-pressure $\mathrm{Hg}(\mathrm{Ar})$ calibration lamp. Intensities have been normalized to the peak intensity.

profile (Fig. 3a). The shape of the antisymmetric curve that results from the deviation of the observed line shape from a Lorentzian (Figs $3 \mathrm{~b}$ and $4 \mathrm{a}$ ) is robust; the shape of the deviation curve is independent of either the emitting element or the specific transition responsible for the emission ${ }^{26}$. The ion-broadening parameter $(A)$, which scales as $N_{\mathrm{e}}{ }^{1 / 4}$, where $N_{\mathrm{e}}$ is the plasma electron density, can be determined from the minimum value of the average normalized deviation from a Lorentzian fit (Fig. 4b; ref. 26). In fact, the wavelengths of the maxima, minima and nodes of deviation curves generated from calculated ion-broadened line profiles change only slightly as $A$ varies by as much as a factor of 20 . Only the amplitudes of the normalized deviation curves vary as a function of $A$, and this variation is well modelled by theory ${ }^{26}$. Note that the temperature dependence of $A$ is minor over a large range (5,000-40,000 K; ref. 25), so interpolation to the temperatures observed here presents no difficulty.

From the antisymmetric deviation function shown in Fig. 4b, we can determine $A$, and therefore $N_{\mathrm{e}}$, as a function of the acoustic driving pressure, $P_{\mathrm{a}}$ (which determines the kinetic energy of the collapsing bubble). To make a direct comparison to theory, we have approximated the Debye shielding parameter, $R$, with a value of 0.55 , as has been done in other previous studies ${ }^{26}$. As a result of the weak dependence of the peak asymmetry on $R$ (see Supplementary, Fig. S1) and because $R$ scales only as $N_{\mathrm{e}}^{1 / 6}$, this assumed value for $R$ will introduce minimal error in the determination of $N_{\mathrm{e}}$. An accurate determination of $A$ requires an estimate of the intracavity temperature generated at each $P_{\mathrm{a}}$, which we have done previously ${ }^{19,27}$. With $A$ determined as a function of $P_{\mathrm{a}}$ and the well-known relationship of $A$ for this specific Ar emission line to an absolute $N_{\mathrm{e}}$ (ref. 28), we can now derive $N_{\mathrm{e}}$ from a single sonoluminescing bubble as a function of $P_{\mathrm{a}}$. Summarized in Table 1 are the determined conditions of the SBSL plasma (that is, temperature, plasma electron density and degree of ionization), as well as the properties of the emission lines and the antisymmetric deviation functions, all as a function of $P_{\mathrm{a}}$. The degree of ionization was determined by direct comparison of the previously measured ${ }^{20}$ number density of neutral Ar atoms to the free electron density. Note that the temperatures reported in Table 1 are for heavy particles, and the temperature of the plasma electrons may not necessarily be in local thermodynamic equilibrium because of the transient 
a

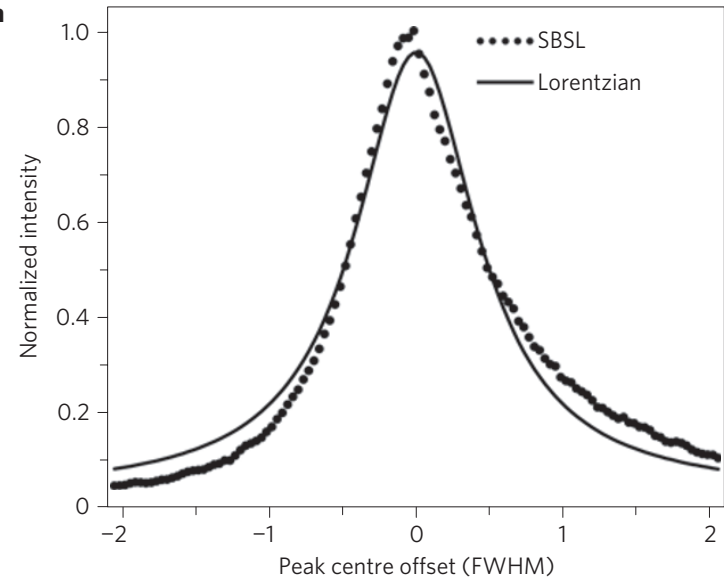

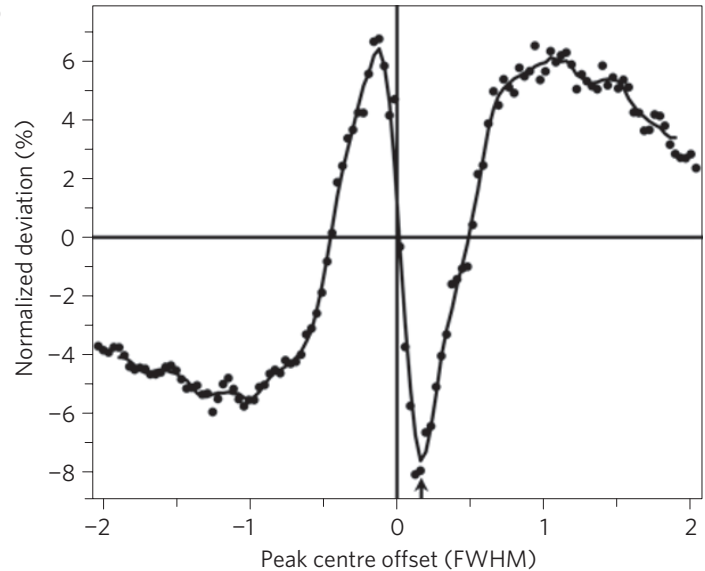

Figure 3 | Line shape and deviation from a Lorentzian fit of the emission from $\operatorname{Ar} 4 p^{2}[3 / 2]$ to $4 s^{2}$ [3/2] states. a, SBSL Ar emission line from a single bubble driven with a $P_{\mathrm{a}}$ of 3.6 bar compared to a least-squares-fit Lorentzian profile. $\mathbf{b}$, The antisymmetric deviation of the Ar emission line from the Lorentzian fit shown in $\mathbf{a}$. The per cent deviation is normalized to the peak intensity of the Lorentzian fit and is plotted as a function of the line peak centre offset in units of FWHM of the Lorentzian. The data are smoothed with a second-degree polynomial regression (Savitzky-Golay filter) using five points to the left and to the right of each point. The arrow at the peak negative deviation marks the deviation used in our analysis.
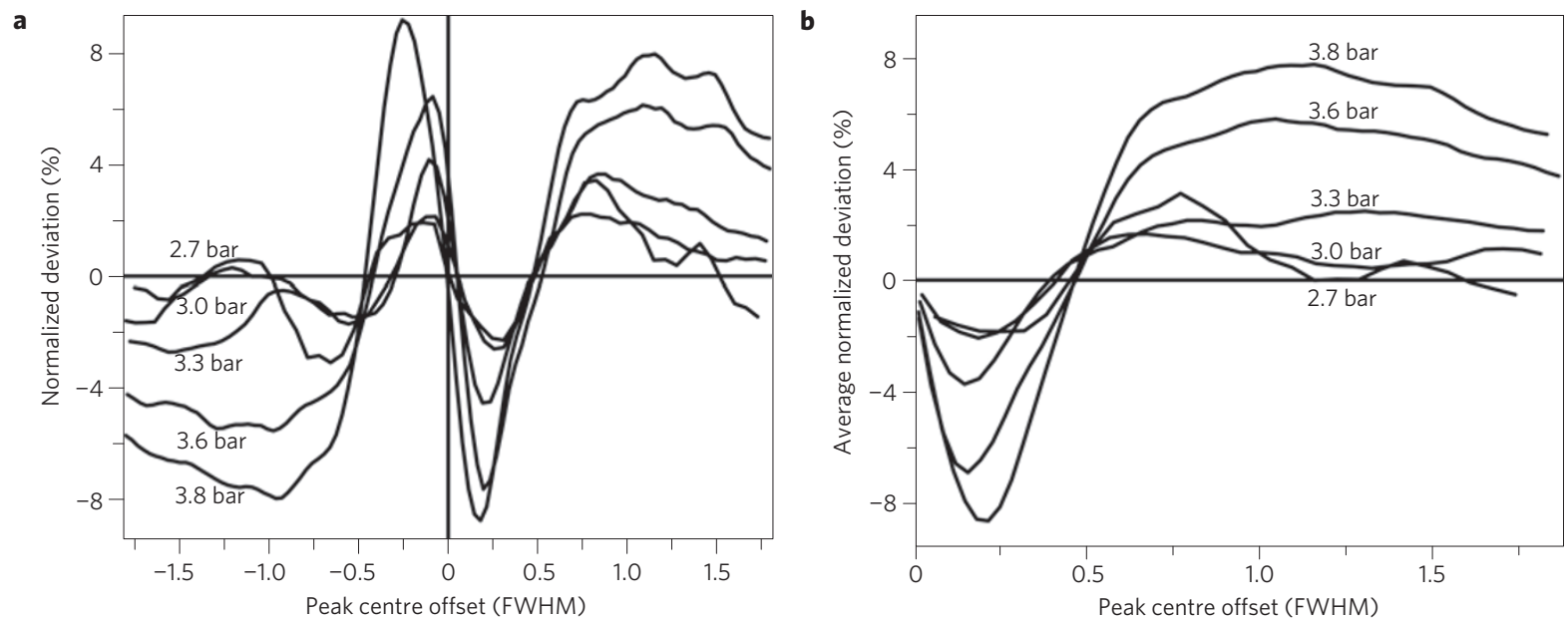

Figure 4 | Deviation from a Lorentzian fit as a function of the acoustic driving pressure, $P_{\mathrm{a}}$, for the emission from $\mathrm{Ar} 4 p^{2}[3 / 2]$ to $4 s^{2}[3 / 2]$ states. a, Dependence of the deviation of the SBSL Ar line profile from a symmetric Lorentzian profile on the $P_{\mathrm{a}}$ used to drive the bubble. All data were smoothed with a second-degree polynomial regression. The deviations were normalized to the peak intensity of the least-squares-fit Lorentzian of each SBSL Ar line profile. $\mathbf{b}$, The antisymmetric deviation function ${ }^{26}$ as a function $P_{\mathrm{a}}$.

Table 1 | Summary of the SBSL Ar line profiles, antisymmetric deviations and plasma conditions as a function of the acoustic driving pressure, $P_{\text {a }}$.

\begin{tabular}{llllllll}
$\boldsymbol{P}_{\mathrm{a}}($ bar) & $\boldsymbol{x}_{\mathrm{c}}(\mathrm{nm})$ & $\boldsymbol{w}(\mathrm{nm})$ & $\boldsymbol{T}_{\text {Ar }}(\mathrm{K})$ & $\Delta_{\text {amp }}(\%)$ & $\boldsymbol{A}$ & $\boldsymbol{N}_{\mathrm{e}}\left(\mathrm{cm}^{-\mathbf{3}}\right)$ & $\boldsymbol{\alpha}$ \\
\hline 2.7 & 764.26 & 0.78 & 7,000 & -1.9 & 0.093 & $4 \times 10^{17}$ \\
3.0 & 764.37 & 0.95 & 10,000 & -2.1 & 0.10 & $1 \times 10^{18}$ \\
3.3 & 764.43 & 1.21 & 13,000 & -3.8 & 0.20 & $2 \times 10^{19}$ & $8 \times 10^{-4(\mathrm{p})}$ \\
3.6 & 764.48 & 1.47 & 15,000 & -6.9 & 0.45 & $5 \times 10^{20}$ \\
3.8 & 764.44 & 1.55 & 16,000 & -8.7 & 0.69 & $4 \times 10^{21}$ \\
\hline
\end{tabular}

The properties of the lines $\left(x_{\mathrm{C}}=\right.$ line centre; $w=$ line full-width at half-maximum (FWHM)) were determined from the least-squares Lorentzian fit. $T_{\mathrm{Ar}}$ is the SBSL temperature determined from relative intensities of the Ar lines. $\Delta_{\mathrm{amp}}$ is the amplitude of the minimum in the antisymmetric deviation function shown in Fig. $4 \mathrm{~b}$. $A$ is the ion-broadening parameter. $N_{\mathrm{e}}$ is the plasma electron density derived from $A$, which scales with $N_{e}^{1 / 4}$. $\alpha$ is the degree of ionization of the plasma. The superscripts in the last column denote whether the plasma can be treated as a perfect (that is, non-interacting) gas ( $p$ ) or must be treated as an imperfect (that is, interacting) gas (i). Note that the SBSL plasma generated at $P_{\mathrm{a}}=3.6$ bar is only weakly imperfect. The estimated error in the per cent deviation amplitude is $\pm 10 \%$, and that of the ion-broadening parameter and plasma electron density is $\pm 18 \%$.

nature of the cavitation event; nonetheless, because of the high pressures also created during SBSL, the heavy-particle temperature is a reasonable first approximation to the electron temperature ${ }^{20}$. The temperature determined at the highest $P_{\mathrm{a}}$ should, however, be considered a lower bound resulting from effective depopulation of the Ar atom excited states by ionization, as reflected in the minimal increase in temperature relative to that at $P_{\mathrm{a}}=3.6$ bar. In addition, the use of Fermi-Dirac quantum statistics at high $P_{\mathrm{a}}$ is necessary for 
describing the plasma thermodynamics, as the criterion for treating the SBSL plasma as a perfect (that is, non-interacting) gas (that is, $N \ll\left(k_{\mathrm{B}} T / Z^{3} e^{2}\right)^{3}$; where $N$ is the particle number density, $k_{\mathrm{B}}$ is the Boltzmann constant, $T$ is the plasma temperature, $Z$ is the average particle charge and $e$ is the elementary charge $)^{29}$ is met only at low effective $P_{\mathrm{a}}$. Here, the crossover from classical Boltzmann statistics to quantum statistics occurs at $P_{\mathrm{a}} \sim 3.6$ bar (see Table 1 ).

As shown in Table 1, the temperature, plasma electron density and degree of ionization markedly increase with increasing acoustic driving pressure. For the maximum $P_{\mathrm{a}}$ used here (which is limited only by the line broadening of the $\mathrm{Ar}$ emission), $N_{\mathrm{e}}$ exceeds $10^{21} \mathrm{~cm}^{-3}$. Astoundingly, this plasma electron density is comparable to that generated by the Lawrence Livermore National Laboratory Nova laser $(1.8 \mathrm{~kJ}$ in $1 \mathrm{~ns}$ at $527 \mathrm{~nm})$ in inertial confinement fusion experiments on a polyethylene target ${ }^{7}$. At the highest $P_{\mathrm{a}}$ used here, the Ar atom number density inside the bubble ${ }^{20}$ is comparable to the maximum plasma electron density, thus providing indirect evidence that some Ar atoms are multiply ionized. Indeed, the ultimate conditions measured here provide evidence that some $\mathrm{Ar}$ atoms are triply ionized. Note that the energy required to move an electron from the valence shell of $\mathrm{Ar}^{2+}$ to vacuum is $40.7 \mathrm{eV}$, and the total energy required for the reaction $\mathrm{Ar} \rightarrow \mathrm{Ar}^{3+}+3 e^{-}$ is $84 \mathrm{eV}$ (that is, $\sim 10^{6} \mathrm{~K}$ if ionization were thermal). The actual ionization potentials will be several electronvolts lower in a dense plasma because of Coulomb interactions ${ }^{29}$, but these energies are nonetheless remarkable.

Although reduced ionization potentials resulting from Coulomb interactions may at least partially explain the high degree of ionization, other factors that are less amenable to quantification may also contribute: for example, the possible formation of a plasma double layer (that is, charge separation resulting from differences in particle mass) because of an inward-focusing shock wave launched from the imploding bubble interface ${ }^{29}$. The effect of the resulting charge separation on the neutral Ar atom emission line profiles will depend on the spatial location of the atoms during photon emission. Similar to plasma double layers, a Debye sheath may form at the interfacial region of the bubble owing to the liquid acting as a solid boundary relative to the ionized gas. The effect of this mechanism on the line profiles will again depend on the spatial location of the radiating atoms within the sonoluminescing region. Although a material spatial map of the sonoluminescing volume is still lacking, it can be reasonably expected that the radiating atoms will occupy regions that are ion rich owing to their very similar masses.

It was recently reported that emission from noble-gas atoms occurs early in the SBSL pulse from $\mathrm{H}_{2} \mathrm{SO}_{4}$ and has a lifetime that is a small fraction of the total duration of emitted light ${ }^{30}$. Timeaveraged spectra could therefore be a convolution of time-varying Ar line profiles. Given the experimental data we report here, it will be of great interest to determine the SBSL plasma properties as a function of time during the emission flash. The implications for the ultimate conditions that may be generated during acoustic cavitation in exotic liquids are remarkable. As bubble implosion proceeds, there is experimental evidence of the formation of an optically opaque core ${ }^{19}$. The interior plasma at the core of the collapsing bubble, which is not visible by emission spectroscopy, must have conditions that exceed, perhaps markedly, temperature and electron densities measured for the outer emitting surface shown in Table 1 . The plasma conditions within the optically opaque core of a collapsing bubble may be truly extraordinary.

Received 16 December 2009; accepted 10 May 2010; published online 27 June 2010

\section{References}

1. Moss, W. C., Clarke, D. B. \& Young, D. A. Calculated pulse widths and spectra of a single sonoluminescing bubble. Science 276, 1398-1401 (1997).
2. Bass, A., Ruuth, S. J., Camara, C., Merriman, B. \& Putterman, S. Molecular dynamics of extreme mass segregation in a rapidly collapsing bubble. Phys. Rev. Lett. 101, 234301 (2008).

3. Putterman, S. J. \& Weninger, K. R. Sonoluminescence: How bubbles turn sound into light. Annu. Rev. Fluid Mech. 32, 445-476 (2000).

4. Brenner, M. P., Hilgenfeldt, S. \& Lohse, D. Single-bubble sonoluminescence. Rev. Mod. Phys. 74, 425-484 (2002).

5. Suslick, K. S. \& Flannigan, D. J. Inside a collapsing bubble: Sonoluminescence and the conditions during cavitation. Annu. Rev. Phys. Chem. 59, 659-683 (2008).

6. Flannigan, D. J. \& Suslick, K. S. Plasma line emission during single-bubble cavitation. Phys. Rev. Lett. 95, 044301 (2005).

7. Ress, D. et al. Measurement of laser-plasma electron density with a soft X-ray laser deflectometer. Science 265, 514-517 (1994).

8. Barber, B. P. \& Putterman, S. J. Observation of synchronous picosecond sonoluminescence. Nature 352, 318-320 (1991).

9. Suslick, K. S. Sonochemistry. Science 247, 1439-1445 (1990)

10. Weninger, K. R., Barber, B. P. \& Putterman, S. J. Pulsed Mie scattering measurements of the collapse of a sonoluminescing bubble. Phys. Rev. Lett. 78, 1799-1802 (1997).

11. Matula, T. J. Inertial cavitation and single-bubble sonoluminescence. Phil. Trans. R. Soc. Lond. A 357, 225-249 (1999).

12. Taleyarkhan, R. P. et al. Evidence for nuclear emissions during acoustic cavitation. Science 295, 1868-1873 (2002).

13. Taleyarkhan, R. P. et al. Nuclear emissions during self-nucleated acoustic cavitation. Phys. Rev. Lett. 96, 034301 (2006).

14. Naranjo, B. Comment on 'Nuclear emissions during self-nucleated acoustic cavitation'. Phys. Rev. Lett. 97, 149403 (2006).

15. Reich, E. S. Is bubble fusion simply hot air? Nature doi:10.1038/news060306-2 (2006).

16. Shapira, D. \& Saltmarsh, M. Nuclear fusion in collapsing bubbles-Is it there? An attempt to repeat the observation of nuclear emissions from sonoluminescence. Phys. Rev. Lett. 89, 104302 (2002).

17. Camara, C. G., Hopkins, S. D., Suslick, K. S. \& Putterman, S. J. Upper bound for neutron emission from sonoluminescing bubbles in deuterated acetone. Phys. Rev. Lett. 98, 064301 (2007).

18. Geisler, R., Schmidt-Ott, W. D., Kurz, T. \& Lauterborn, W. Search for neutron emission in laser-induced cavitation. Europhys. Lett. 66, 435-440 (2004).

19. Flannigan, D. J. \& Suslick, K. S. Plasma formation and temperature measurement during single-bubble cavitation. Nature 434, 52-55 (2005).

20. Flannigan, D. J., Hopkins, S. D., Camara, C. G., Putterman, S. J. \& Suslick, K. S. Measurement of pressure and density inside a single sonoluminescing bubble. Phys. Rev. Lett. 96, 204301 (2006).

21. Didenko, Y. T. \& Suslick, K. S. The energy efficiency of formation of photons, radicals and ions during single-bubble cavitation. Nature 418, 394-397 (2002).

22. Storey, B. D. \& Szeri, A. J. Water vapour, sonoluminescence and sonochemistry. Proc. R. Soc. Lond. A 456, 1685-1709 (2000).

23. Flannigan, D. J., Hopkins, S. D. \& Suslick, K. S. Sonochemistry and sonoluminescence in ionic liquids, molten salts, and concentrated electrolyte solutions. J. Organomet. Chem. 690, 3513-3517 (2005).

24. Hilgenfeldt, S., Grossmann, S. \& Lohse, D. A simple explanation of light emission in sonoluminescence. Nature 398, 402-405 (1999).

25. Griem, H. R. Spectral Line Broadening by Plasmas (Academic, 1974)

26. Jones, D. W., Wiese, W. L. \& Woltz, L. A. Ion broadening of Ar I lines in a plasma. Phys. Rev. A 34, 450-456 (1986).

27. McNamara, W. B., Didenko, Y. T. \& Suslick, K. S. Sonoluminescence temperatures during multi-bubble cavitation. Nature 401, 772-775 (1999).

28. Milosavljević, V. \& Djeniže, S. Ion contribution to the prominent Ne I, Ar I and Kr I spectral line broadening. Astron. Astrophys. 398, 1179-1184 (2003).

29. Zel'dovich, Ya., Raizer, B. \& Yu, P. Physics of Shock Waves and High-Temperature Hydrodynamic Phenomena (Dover Publications, 2002).

30. Chen, W. Z., Huang, W., Liang, Y., Gao, X. X. \& Cui, W. C. Time-resolved spectra of single-bubble sonoluminescence in sulfuric acid with a streak camera. Phys. Rev. E 78, 035301 (2008).

\section{Acknowledgements}

This work was supported by the National Science Foundation. We thank S. Putterman and N. Eddingsaas for useful discussions.

\section{Author contributions}

All authors contributed extensively to the work presented in this paper.

\section{Additional information}

The authors declare no competing financial interests. Supplementary information accompanies this paper on www.nature.com/naturephysics. Reprints and permissions information is available online at http://npg.nature.com/reprintsandpermissions. Correspondence and requests for materials should be addressed to K.S.S. 